\title{
Optimal generation for wind-thermal power plant systems with multiple fuel sources
}

\author{
Phan Nguyen Vinh ${ }^{1}$, Bach Hoang Dinh ${ }^{2}$, Van-Duc Phan ${ }^{3}$, Hung Duc Nguyen ${ }^{4}$, Thang Trung Nguyen ${ }^{5}$ \\ ${ }^{1}$ Faculty of Cinema and Television, The University of Theatre and Cinema Ho Chi Minh City, Vietnam \\ 2,5 Power System Optimization Research Group, Faculty of Electrical and Electronics Engineering, Ton Duc Thang \\ University, Ho Chi Minh City, Vietnam \\ ${ }^{3}$ Faculty of Automobile Technology, Van Lang University, Ho Chi Minh City, Vietnam \\ ${ }^{4}$ Faculty of Electrical and Electronics Engineering, Ho Chi Minh City University of Technology, Vietnam National \\ University Ho Chi Minh City, Vietnam
}

\section{Article Info}

Article history:

Received May 29, 2020

Revised Sep 10, 2020

Accepted Oct 7, 2020

\section{Keywords:}

Cuckoo search algorithm

Multiple fuel sources

Thermal power plant

Total fossil fuel cost

Wind power plant

\begin{abstract}
In this paper, the combined wind and thermal power plant systems are operated optimally to reduce the total fossil fuel cost (TFFC) of all thermal power plants and supply enough power energy to loads. The objective of reducing TFFC is implemented by using antlion algorithm (ALA), particle swarm optimization (PSO) and Cuckoo search algorithm (CSA). The best method is then determined based on the obtained TFFC from the three methods as dealing with two study cases. Two systems with eleven units including one wind power plant (WPP) and ten thermal power plants are optimally operated. The two systems have the same characteristic of MFSs but the valve loading effects (VLEs) on thermal power plants are only considered in the second system. The comparisons of TFFC from the two systems indicate that CSA is more powerful than ALA and PSO. Furthermore, CSA is also superior to the two methods in terms of faster search process. Consequently, CSA is a powerful method for the problem of optimal generation for wind-thermal power plant systems with consideration of MFSs from thermal power plants.
\end{abstract}

This is an open access article under the CC BY-SA license.

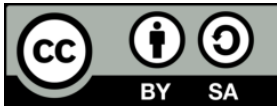

\section{Corresponding Author:}

Thang Trung Nguyen

Power System Optimization Research Group

Faculty of Electrical and Electronics Engineering

Ton Duc Thang University

19 Nguyen Huu Tho street, Tan Phong ward, District 7, Ho Chi Minh City, Vietnam

Email: nguyentrungthang@tdtu.edu.vn

\section{INTRODUCTION}

The main text format consists of a flat left-right columns on A4 paper (quarto). The margin text from the In power system operation, the main target of operating thermal power plants (TPPs) is to determine the most appropriate active power generation of each thermal power plant (TPP) to reduce TFFC as much as possible [1-3]. The fact that fossil fuel sources (FFSs) will be exhausted in future and its cost will increase. So, the optimal use plan of the fuels can enable to last the use time of the sources and power system will be more stable and work with high reliability. The purpose of using FFSs with lower cost and long time is encouraged in power systems $[4,5]$.

The problem of minimizing TFFC from TPPs was concerned in many recent decays. This problem was called economic load dispatch (ELD) and mathematical modeled by the presence of objective function and constraints such as limits of generation and active power balance [6-8]. Some first ELD problems 
considered only single fuel source (SFS) for each TPP and only the minimum and maximum power generation of the sole SFS was considered. More complicated problems considered total power loss in transmission lines and ignored other complicated constraints regarding TPPs such as reserve fuel source limits and VLEs [9, 10]. There many algorithm types have been applied for the problem with SFS such as deterministic algorithms and metaheuristic algorithms. Among the two algorithm groups, the second group was more widely and successfully applied. The first algorithm group consists of Lagrange method [11], Newton method [12, 13], dynamic programming [14] and gradient search method [15]. The second group includes PSO [16-17], genetic algorithm (GA) [18-20], differential evolution (DE) [21], fractal search algorithm (FSA) [22], teaching learning optimization algorithm (TLOA) [23], bee colony algorithm (BCA) [24], crow optimization algorithm (COA) [25] and hybrid algorithm (HA) [26]. Deterministic methods had an advantage of using a low iteration number and reaching the same results for different implementations. However, they suffering from taking partial derivative with respect to control variables before executing an iterative algorithm. Hence, they could not be applied for solving ELD problems containing non-differentiable functions. On the contrary, metaheuristic algorithms did not suffer from the shortcoming of the deterministic algorithms and they could deal with nondifferentiable function and complicated problem. But the metaheuristic algorithms had the same disadvantages of falling into local search zones with local optimal solution or a nearby global solution with worse quality than the best solutions.

As ELD problem is more complicated by considering much complicated fossil fuel cost function (FFCF) and constraints. VLEs were considered during power increasing or decreasing process of thermal power plants [26]. A complicated FFCF considered MFSs for burning fuel and driving steam or gas turbines [27]. The combination of VLEs and MFSs was implemented and the solution of the problem was found in the study [28]. The model with both VLEs and MFSs is the most complicated FFCF in ELD problems. However, all the considered problems did not consider renewable energies like wind power plants or photovoltaic systems. Nowadays, wind power plants (WPPs) can produce a very high power and supply electricity to loads via transmission power network. There were studies combining the wind power plant and thermal power plants [29-30] for determining TFFC minimization. In the study [29], wind velocity was modeled by a probability function and its power was dependent on the function. Thermal power plants were in charging of producing a remaining power after wind power plant can supply its power to loads. The study mainly introduced the change of wind velocity or considered the uncertainty of wind velocity rather than minimizing total fuel cost of TPPs. In the study [30], PSO and bat algorithm (BA) were applied to optimize power generation for TPPs and WPPs. Two systems with 7 plants and 16 plants using SFS were employed in the study. The demonstration was that power generation from WPP could reduce TFFC of all TPPs and BA was superior to PSO in terms of reducing TFFC.

In this paper, WPPs and TPPs are combined to produce electricity to loads in which MFSs is considered in TPPs to produce electricity. In addition, the objective function becomes more complicated since VLEs is considered during the generation process of the TPPs. Two systems with eleven plants including one WPP and ten TPPs are taken into account. For reaching the optimal generation of the plants, three methods including antlion algorithm (ALA) [31], particle swarm optimization (PSO) [32] and cuckoo search algorithm (CSA) [33] are implemented. The novelty and contribution of the paper is as follows:

- Combine WPPs and TPPs where TPPs consider MFSs and VLEs

- Compare the performance of PSO, ALA and CSA

- Provide optimal solutions for reducing TFFC for the combined system

Other parts of the paper are as follows: Section 2 describes objective function and constraints of the combined system. Section 3 present Cuckoo search algorithm. Section 4 shows the result comparisons between CSA and two other methods. Section 5 shows the conclusions.

\section{THE PROBLEM FORMULATION}

\subsection{Objective function of the ELD problem with single fuel option}

In optimal operation of the combined system, power generated by WPPs and TPPs is supplied to loads in which the cost of power from WPPs is supposed to be a constant and it is much less than the cost of power from TPPs. So, the objective is to use all power from WPPs while the cost from TPPs. The objective is as follows:

$$
\text { Minimize } T F F C=\sum_{t=1}^{N t} F F C_{t}\left(P_{t}\right)
$$

where $F F C_{t}$ is the fossil fuel cost of the $t$ th TPP; $P t$ is power generation of the $t$ th TPP; $N t$ is the number of TPPs. 
As MFSs is considered, $F F C_{t}$ of the tth TPP is represented as follows:

$$
\text { FFC }_{t}\left(P_{t}\right)=\left\{\begin{array}{cc}
a_{t 1} P_{t}^{2}+b_{t 1} P_{t}+c_{t 1} & \text { for } P_{t, 1, \min } \leq P_{t} \leq P_{t, 1, \max } \\
a_{t 2} P_{t}^{2}+b_{t 2} P_{t}+c_{t 2} & \text { for } P_{t, 2, \min } \leq P_{t} \leq P_{t, 2, \max } \\
\ldots & \text { for } P_{t, M, \min } \leq P_{t} \leq P_{t, M, \max }
\end{array}\right.
$$

where $M$ is the number of fuels; $P_{t, M, \min }$ and $P_{t, M, \max }$ are the lower bound and upper bound of power generation of the $t$ th TPP for the Mth fuel.

As considering the VLEs in the generation process, FFCt of the tth TPP is represented as follows:

$$
F F C_{t}\left(P_{t}\right)=\left\{\begin{array}{cc}
a_{t 1} P_{t}^{2}+b_{t 1} P_{t}+c_{t 1}+\left|\delta_{t 1} \times \sin \left(\varepsilon_{t 1} \times\left(P_{t, \text { min }}-P_{t}\right)\right)\right| & \text { for } P_{t, 1, \text { min }} \leq P_{t} \leq P_{t, 1, \max } \\
a_{t 2} P_{t}^{2}+b_{t 2} P_{t}+c_{t 2}+\left|\delta_{t 2} \times \sin \left(\varepsilon_{t 2} \times\left(P_{t, \text { min }}-P_{t}\right)\right)\right| & \text { for } P_{t, 2, \text { min }} \leq P_{t} \leq P_{t, 2, \text { max }} \\
\cdots & \\
a_{t M} P_{t}^{2}+b_{t M} P_{t}+c_{t M}+\left|\delta_{t M} \times \sin \left(\varepsilon_{t M} \times\left(P_{t, \text { min }}-P_{t}\right)\right)\right| & \text { for } P_{t, M, \text { min }} \leq P_{t} \leq P_{t, M, \text { max }}
\end{array}\right.
$$

The $F F C_{t}$ without and with VLEs is plotted in Figure 1.

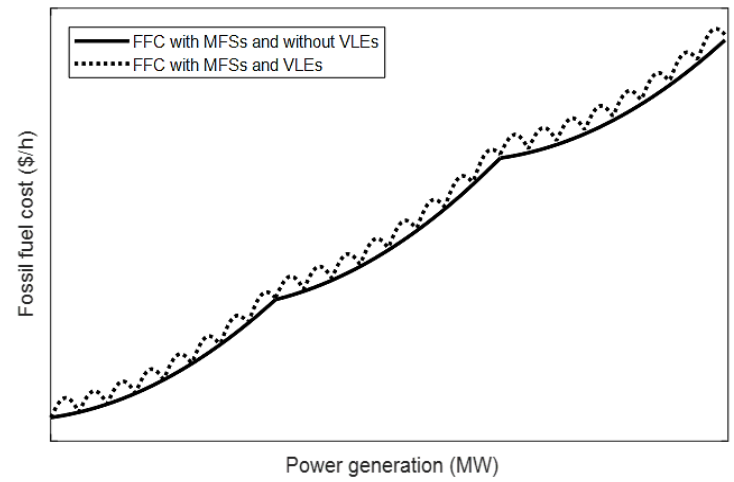

Figure 1. FFC with and without VLEs

\subsection{Power generation of wind turbines}

Wind turbines can produce and supply electricity to loads but their stability is not certain due to the influence of wind velocity. If wind velocity is high, power generation of the wind turbines is high and vice versa. The generation of the wind turbines is determined as follows: [29]:

$$
W P_{w}= \begin{cases}0 & \text { if } V \leq V i \\ W P_{w, r a t e} \frac{V-V i}{V r-V i} & \text { if } V i \leq V \leq V r \\ W P_{w, \text { rate }} & \text { if } V r \leq V \leq V o \\ 0 & \text { else }\end{cases}
$$

where WPw and WPw, rate are Power generation and rated power generation of the wth WPP; V and Vr, are speed of wind, rated speed of wind at the wth WPP; and Vi and Vo are the minimum and maximum speed of wind for power generation.

\subsection{Constraints of the problem}

\subsubsection{Constraints of WPPs}

Wind power is dependent on values of velocity. So, as the velocity has the lowest value, the power is minimum and as the velocity has the highest value, the power is maximum. As a results, limits of wind power are as follows: 


$$
W P_{w, \min } \leq W P_{w} \leq W P_{w, \max } ; w=1, \ldots, N w
$$

where $W P_{w}$ is power generation of the $w t h$ WPP; $W P_{w, \min }$ and $W P_{w, \min }$ are the lower bound and upper bound of power generation of the $w t h$ WPP; and $N w$ is the number of WPPs.

\subsubsection{Constraints of TPPs}

Active power generation limits: In the problem, the fossil fuel sources at TPPs are supposed to be plentiful and unlimited. However, the power generation of each TPP is constrained due to the limits of gas/steam turbines and generators. The generation restriction is described as follows:

$$
P_{t, \min } \leq P_{t} \leq P_{t, \max }
$$

In the constraint, $P_{t, \min }$ and $P_{t, \max }$ are the minimum and maximum active power generation of the $t$ th TPP. Because the TPPs use MFSs, $P_{t, \text { min }}$ and $P_{t, \text { max }}$ are, respectively, equal to $P_{t, 1, \text { min }}$ and $P_{t, M, \max }$, which are shown in (2) and (3).

\subsubsection{Constraints of system}

The power system constrains the balance of power between supply side and consume side. If the balance is achieved, the frequency of the system can be stable and system is working stably. The supply side consists of TPPs and WPPs where consume side is load demand. The balance constraint is as follows:

$$
\sum_{t=1}^{N t} P_{t}+\sum_{w=1}^{N w} W P_{w}=P_{d e m a n d}
$$

where $P_{\text {demand }}$ is the active power of all loads in the system.

\section{CUCKOO SEARCH ALGORITHM (CSA)}

\subsection{Lévy flights}

Lévy flights is a power search technique of CSA thank to the use of an infinite step size by using Lévy distribution. The technique is used to update new solutions as shown in the following equation:

$$
\operatorname{Sol}_{s}^{\text {new }}=\operatorname{Sol}_{s}+\varepsilon . \text { Levy; } s=1, \ldots, N_{p o}
$$

where $\varepsilon$ is scale factor; Levy is Lévy distribution [33]; and Npo is population size.

\subsection{Mutation technique}

Mutation technique is employed in CSA to update new solutions for the second time. However, the technique does not update the whole population but it selects solutions based on the comparison condition between a random number cs and mutation factor MF. The technique is shown as follows:

$$
\mathrm{Sol}_{s}^{\text {new }}= \begin{cases}\operatorname{Sol}_{s}+c .\left(\mathrm{Sol}_{r p 1}-\mathrm{Sol}_{r p 2}\right) & \text { if } c_{s}<M F \\ \operatorname{Sol}_{s} & \text { otherwise }\end{cases}
$$

where $S o l_{r p 1}$ and $\operatorname{Sol}_{r p 2}$ are picked solutions by using randomization.

\section{NUMERICAL RESULTS}

In this section, PSO, ALA and CSA are executed for reaching the optimal solutions of two test systems. Each method is run fifty trials on Matlab platform and a PC with processor of $2.2 \mathrm{GHz}$ and RAM of 4.0GB. The detail of simulation result is presented in the two following sections.

\subsection{The first wind-thermal power plant system}

In this section, the data of ten TPPs with MFSs and without VLEs is taken from [34] while the data of WPP is taken from [35]. The rated speed of wind and the rated power of the WPP is $15 \mathrm{~m} / \mathrm{s}$ and $120 \mathrm{MW}$. 
The wind speed at the scheduling hour is $14 \mathrm{~m} / \mathrm{s}$ and the power generation of the WPP is $108 \mathrm{MW}$. The TPPs and the WPP supply electricity to a $2400 \mathrm{MW}$ load. In order to get results for PSO, ALA and CSA, Npo and Nit (which is the number of iterations) are respectively set to 20 and 100 for PSO and ALA, and 10 and 100 for CSA. The results are summarized in Figures 2 and 3. Figure 2 shows that CSA can reach the smallest cost with $\$ 437.7$ while PSO is the worst method with the highest cost of $\$ 441.4$. Similarly, CSA and PSO are the best and the worst methods with the smallest and highest maximum cost. The comparisons of mean cost of 50runs can indicate the best stability of CSA since that of the method is the smallest. Figure 3 can confirm the best stability of CSA one more time since all runs of CSA have much less cost than those of PSO and ALA.

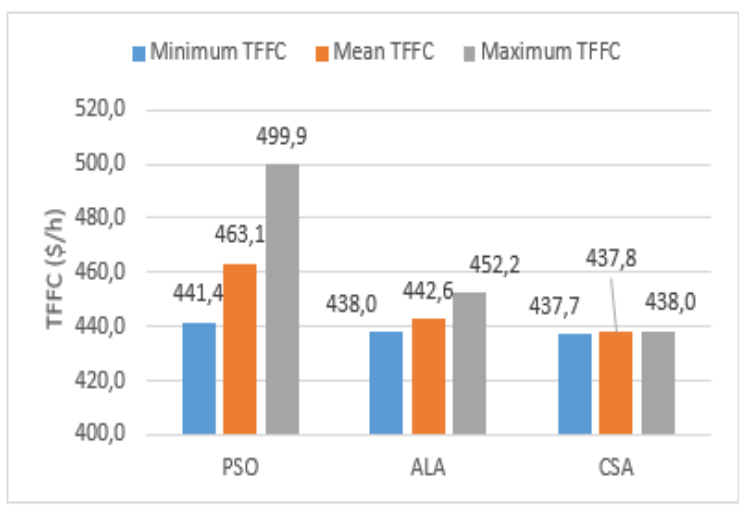

Figure 2. Comparisons of result for the first system

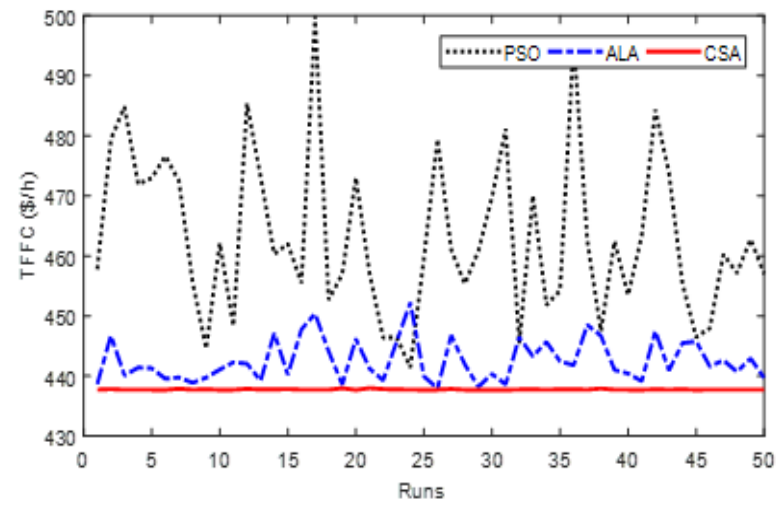

Figure 3. TFFC comparisons of 50 trial runs for the first system

\subsection{The second wind-thermal power plant system}

In this section, the data of ten TPPs with MFSs and VLEs is taken from [35] while the data of WPP is taken from [36]. The rated speed of wind and the rated power of the WPP is $15 \mathrm{~m} / \mathrm{s}$ and $80 \mathrm{MW}$. The wind speed at the scheduling hour is $14.5 \mathrm{~m} / \mathrm{s}$ and the power generation of the WPP is $76 \mathrm{MW}$. The TPPs and the WPP supply electricity to a $2700 \mathrm{MW}$ load.

For running PSO, ALA and CSA, Npo and Nit are respectively set to 20 and 200 for PSO and ALA, and 10 and 200 for CSA. The Figures 4 and 5 show the best performance of CSA because CSA can reach the smallest minimum, mean and maximum cost. ALA is the second best method whereas PSO is still the worst performance method. TFFC of 50 runs from CSA is approximately lied on a line while that of PSO and ALA have the high fluctuations. The analysis can lead to a conclusion that CSA is superior to PSO and ALA for the system with MFSs, VLEs and wind power plants. Optimal solutions of the two systems are shown Table A1 in Appendix.

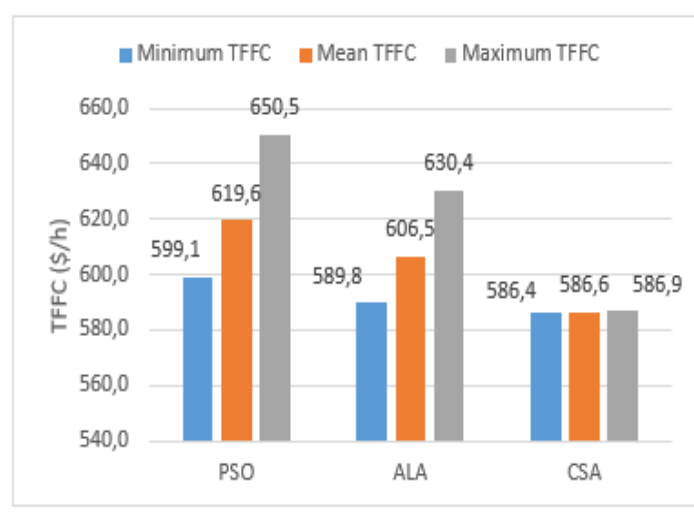

Figure 4. Comparisons of result for the second system

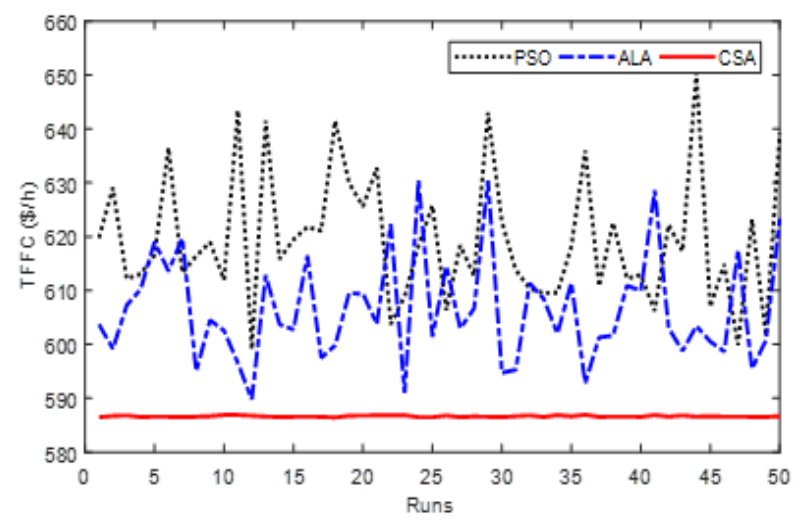

Figure 5. TFFC comparisons of 50 trial runs for the second system 


\section{CONCLUSION}

In this paper, PSO, ALA and CSA solved two wind-thermal power plant systems where TPPs used multiple fuel sources to generate electricity to loads. The two system had the same number of power plants, ten TPPs and one WPP in which the first system did not consider valve loading effects on TPPs during the power increase or decrease process but the second system did. The result comparison indicated that CSA was more robust than PSO and ALA in reaching the optimal power generation and the search stability. CSA could reach the smallest minimum, mean and maximum cost among three executed methods. In addition, the cost from fifty runs of CSA was approximately lied on a line without fluctuations whilst the cost of PSO and ALA had high fluctuations. Consequently, CSA is recommended as a powerful search algorithm for the optimal generation of wind-thermal power plant systems.

\section{APPENDIX}

Table A1. Optimal generation of the two systems found by PSO, ALA and CSA methods

\begin{tabular}{|c|c|c|c|c|c|c|}
\hline \multirow{2}{*}{$P_{t}(\mathrm{MW})$} & \multicolumn{3}{|c|}{ System 1} & \multicolumn{3}{|c|}{ System 2} \\
\hline & PSO & ALA & CSA & PSO & ALA & CSA \\
\hline$P_{1}$ & 168.9 & 182.7 & 179.8 & 207.8 & 195.3 & 208.4 \\
\hline$P_{2}$ & 208.6 & 197.3 & 196.8 & 303.2 & 273.6 & 271.3 \\
\hline$P_{3}$ & 220.9 & 239.9 & 239.6 & 265.0 & 237.6 & 237.5 \\
\hline$P_{4}$ & 220.2 & 232.2 & 230.4 & 298.8 & 244.9 & 270.0 \\
\hline$P_{5}$ & 225.7 & 214.1 & 223.7 & 235.8 & 244.4 & 235.9 \\
\hline$P_{6}$ & 223.5 & 229.2 & 229.8 & 295.2 & 290.9 & 278.1 \\
\hline$P_{7}$ & 261.7 & 236.2 & 235.1 & 234.0 & 234.4 & 238.5 \\
\hline$P_{8}$ & 224.2 & 230.0 & 229.1 & 330.4 & 394.7 & 409.6 \\
\hline$P_{9}$ & 327.0 & 300.8 & 306.5 & 208.8 & 276.6 & 262.8 \\
\hline$P_{10}$ & 211.1 & 229.5 & 221.2 & 244.9 & 231.7 & 211.8 \\
\hline
\end{tabular}

\section{REFERENCES}

[1] Montoya, O. D., Gil-González, W., Grisales-Noreña, L., Orozco-Henao, C., Serra, F., "Economic Dispatch of BESS and Renewable Generators in DC Microgrids Using Voltage-Dependent Load Models," Energies, vol. 12, no. 23, p. 4494, 2019.

[2] Trivedi, I. N., Jangir, P., Bhoye, M., and Jangir, N., "An economic load dispatch and multiple environmental dispatch problem solution with microgrids using interior search algorithm," Neural Computing and Applications, vol. 30 , no. 7 , pp. $2173-2189,2018$

[3] Al-Betar, M. A., Awadallah, M. A., Krishan, M. M., "A non-convex economic load dispatch problem with valve loading effect using a hybrid grey wolf optimizer," Neural Computing and Applications, vol. 32, pp. 12127-12154, 2020.

[4] Gnawali, K., Han, K. H., Geem, Z. W., Jun, K. S., Yum, K. T., "Economic Dispatch Optimization of Multi-Water Resources: A Case Study of an Island in South Korea," Sustainability, vol. 11, no. 21, p. 5964, 2019.

[5] Das, D., Bhattacharya, A., Ray, R. N., "Dragonfly Algorithm for solving probabilistic Economic Load Dispatch problems," Neural Computing and Applications, vol. 32, pp. 3028-3045, 2020.

[6] Ang, S., Leeton, U., Chayakulkeeree, K., Kulworawanichpong, T., "Sine Cosine Algorithm for Optimal Placement and Sizing of Distributed Generation in Radial Distribution Network," GMSARN International Journal vol. 12, pp. 202-212, 2018.

[7] Mmary, E. R., and Marungsri, B., "Integration of Multi-Renewable Energy Distributed Generation and Battery in Radial Distribution Networks," GMSARN International Journal, vol. 12, pp. 194-201, 2018.

[8] Mmary, E. R., and Marungsri, B., "Multiobjective optimization of renewable distributed generation and shunt capacitor for techno-economic analysis using hybrid invasive weeds optimization," GMSARN International Journal, vol. 12, pp. 24-33, 2018.

[9] Kamboj, V. K., Bhadoria, A., and Bath, S. K., "Solution of non-convex economic load dispatch problem for smallscale power systems using ant lion optimizer," Neural Computing and Applications, vol. 28, no. 8, pp. 2181-2192, 2017.

[10] Kamboj, V. K., Bath, S. K., and Dhillon, J. S., "Solution of non-convex economic load dispatch problem using Grey Wolf Optimizer," Neural Computing and Applications, vol. 27, no. 5, pp. 1301-1316, 2016.

[11] Zhou, J., "Solving economic dispatch problem with piecewise quadratic cost functions using Lagrange multiplier theory," In Proc. 3rd ICCTD, pp. 25-27, 2011.

[12] Kaur, N., and Singh, I., "Economic Dispatch Scheduling using Classical and Newton Raphson Method," International Journal of Engineering and Management Research (IJEMR), vol. 5, no. 3, pp. 711-716, 2015.

[13] Lin, C. E., Chen, S. T., and Huang, C. L., “A direct Newton-Raphson economic dispatch," IEEE Transactions on Power Systems, vol. 7, no. 3, pp. 1149-1154, 1992.

[14] Liang, Z. X., and Glover, J. D., "A zoom feature for a dynamic programming solution to economic dispatch including transmission losses," IEEE Transactions on Power Systems, vol. 7, no. 2, pp. 544-550, 1992.

[15] Ray, S., "Economic Load Dispatch Solution using Interval Gradient Method," Advanced Research in Electrical and Electronic Engineering, vol. 1, no. 4, pp. 55-58, 2015. 
[16] Sudhakaran, M., Raj, P. A. D. V., and Palanivelu, T. G., "Application of particle swarm optimization for economic load dispatch problems," In 2007 International Conference on Intelligent Systems Applications to Power Systems, Toki Messe, Niigata, pp. 1-7, 2007.

[17] Aristidis, V., "Particle Swarm Optimization (PSO) techniques solving Economic Load Dispatch (ELD) Problem," Journal of Statistics and Management Systems, vol. 11, no. 4, pp. 761-769, 2008.

[18] Sahay, K. B., Sonkar, A., and Kumar, A., "Economic Load Dispatch Using Genetic Algorithm Optimization Technique," In 2018 International Conference and Utility Exhibition on Green Energy for Sustainable Development (ICUE), Phuket, Thailand, 2018, pp. 1-5.

[19] Kaur, A., Singh, H. P., and Bhardwaj, A., "Analysis of economic load dispatch using genetic algorithm," International Journal of Application or Innovation in Engineering \& Management (IJAIEM), vol. 3, no. 3, pp. 240-246, 2014.

[20] Olakunle, A. O., and Folly, K. A., "Economic Load Dispatch of Power System Using Genetic Algorithm with Valve Point Effect," In International Conference in Swarm Intelligence, vol. 9140, 2015, pp. 276-284.

[21] Dinh, B. H., and Nguyen, T. T., "New solutions to modify the differential evolution method for multi-objective load dispatch problem considering quadratic fuel cost function," In International Conference on Advanced Engineering Theory and Applications, vol. 415, 2016, pp. 598-607.

[22] Phan Van Hong T. and Tran The T., "Economic Dispatch in Microgrid using Stochastic Fractal Search Algorithm," GMSARN International Journal, vol. 11, pp. 102-115, 2017.

[23] Rao, D. S. N. M., and Kumar, N., "Comparisional Investigation of Load Dispatch Solutions with TLBO," International Journal of Electrical and Computer Engineering (IJECE), vol. 7, no. 6, pp. 3246-3253, 2017

[24] Gachhayat, S. K., Dash, S. K., and Ray, P., "Multi Objective Directed Bee Colony Optimization for Economic Load Dispatch With Enhanced Power Demand and Valve Point Loading," International Journal of Electrical \& Computer Engineering (IJECE), vol. 7, no. 5, pp. 2382-2391, 2017

[25] Spea, S. R., "Solving practical economic load dispatch problem using crow search algorithm," International Journal of Electrical and Computer Engineering (IJECE), vol. 10, no. 4, pp. 3431-3440, 2020.

[26] Rajesh, K., and Visali, N., "Hybrid method for achieving Pareto front on economic emission dispatch," International Journal of Electrical and Computer Engineering (IJECE), vol. 10, no. 4, pp. 3358-3366, 2020.

[27] Basu, M., "Modified particle swarm optimization for nonconvex economic dispatch problems," International Journal of Electrical Power \& Energy Systems, vol. 69, pp. 304-312, 2015.

[28] Balamurugan, R., Subramanian, S., "Hybrid integer coded differential evolution-dynamic programming approach for economic load dispatch with multiple fuel options," Energy Conversion and Management, vol. 49, no. 4, pp. 608-614, 2008.

[29] Chiang, C. L., "Improved genetic algorithm for power economic dispatch of units with valve-point effects and multiple fuels," IEEE transactions on power systems, vol. 20, no. 4, pp. 1690-1699, 2005.

[30] Hetzer, J., David, C. Y., and Bhattarai, K., "An economic dispatch model incorporating wind power," IEEE Transactions on energy conversion, vol. 23, no. 2, pp. 603-611, 2008.

[31] Jose, J. T., "Economic load dispatch including wind power using Bat Algorithm," In 2014 International Conference on Advances in Electrical Engineering (ICAEE), Vellore, 2014, pp. 1-4.

[32] Amaireh, A. A., Al-Zoubi, A. S., and Dib, N. I., "The optimal synthesis of scanned linear antenna arrays," International Journal of Electrical and Computer Engineering (IJECE), vol. 10, no. 2, pp. 1477-1484, 2020.

[33] Abdul-Adheem, W. R., "An enhanced particle swarm optimization algorithm," International Journal of Electrical and Computer Engineering (IJECE), vol. 9, no. 6, pp. 4904-4907. 2019.

[34] Paul, K., and Kumar, N., "Cuckoo Search Algorithm for Congestion Alleviation with Incorporation of Wind Farm," International Journal of Electrical \& Computer Engineering (IJECE), vol. 8, no. 6, pp. 4871-4879, 2018.

[35] Zhang, H., Yue, D., Xie, X., Dou, C., Sun, F., "Gradient decent based multi-objective cultural differential evolution for short-term hydrothermal optimal scheduling of economic emission with integrating wind power and photovoltaic power," Energy, vol. 122, pp. 748-766, 2017.

[36] Lee, K. Y., Sode-Yome, A., Park, J. H., "Adaptive Hopfield neural networks for economic load dispatch," IEEE transactions on power systems, vol. 13, no. 2, pp. 519-526, 1998. 